\title{
Enhancing the Accuracy of Indoor Positioning by Using Multiple Bluetooth Low Energy Beacon Devices
}

\author{
Huai-Hsiang Chang, Yen-Ching Chu, Yen-Bin Huang, Tzu-Yuan Lin and Ming-Fong Tsai* \\ Department of Information Engineering and Computer Science, Feng Chia University, Taichung, Taiwan
}

\begin{abstract}
In recent years indoor positioning techniques based on the received signal strength indicator (RSSI) signal of Bluetooth Low Energy beacon devices (BLE) has become an interesting research topic. However, this beacon device has many problems in terms of positioning accuracy. RSSI emitted from these devices is not stable and the mathematical model of the current system does not produce highly accurate results. To enhance positioning accuracy, we propose to use multiple beacon devices at the same corner and filter out the RSSI deviation, which can reduce the probability of unstable RSSI. We also propose a mathematical model to calculate the exact location of the user in the system. The obtained results show that our method significantly improved the correct estimation rate of the positioning method.
\end{abstract}

Keywords: Received Signal Strength Indicator, Bluetooth Low Energy, Beacon.

\section{Introduction}

Real-time Indoor Positioning Systems (IPS) have undergone rapid development and become critical techniques for a vast number of indoor applications. The traditional Global Positioning System (GPS) can no longer ensure the sufficient accuracy to locate objects inside buildings due to its 10-metre location accuracy and the strong attenuation of the GPS signal in indoor environments. Therefore, new technologies like GPS systems are required that can help accurately locate objects inside buildings. There are several solutions for indoor positioning systems, such as Bluetooth [1-3], Wi-Fi [4] and Ultrasound [5] sensor systems. However, these systems face some limitations, including high cost, high-energy consumption and the received signal is often unstable due to the noise of indoor environment, which leads to low accuracy.

Recently, the development of smart handheld devices, especially smartphones, which can receive RSSI signal through Bluetooth connectivity, provided an effective solution for energy-saving indoor positioning systems. Based on the received RSSI signal, the system can estimate the relative position of the user. Along with the introduction of the Bluetooth Low Energy (BLE) or Bluetooth 4.0 is a spotlight for energy-saving communications. BLE beacon devices have advantages of low cost and ultra-low energy consumption meaning that multiple beacon devices can be arranged in a large building. These beacon devices easily communicate with the user's smartphone via Bluetooth so users can know their location at any time.

Positioning based on the beacon device is not an innovative approach. However, in the past the implementation of this method encountered many challenges. The main problem is that the signal strength is not really correlated with the distance. First, we refer to the paper [2] and implement the methods reported therein. We deployed a single BLE beacon device in every corner of a room and found that this method could not deal with the three-dimensional space unless compression technology was applied or more beacon devices were used. RSSI signals often fluctuated and therefore are not reliable. Second, we refer to the work in [1], where it was reported that at the same distance, the RSSI signal strength of each beacon device is not really the same. Before testing, each beacon device must be calibrated so that each beacon device at the same distance can produce the same signal strength and, thus, test results can be more accurate. Third, we refer to the work in [3]. This study uses a table to store the received signal strength before mapping the distance. We attempted to implement this method but found that this method does not achieve high positioning accuracy. 
This method considers each meter as a unit but in fact, this unit is still too big. If a conversion formula could be customised, this would better reflect the distance.

In this paper, we deploy multiple beacon devices in the same corners to solve the problem of unstable RSSI signal. Using a beacon device will incur RSSI deviation but by using multiple beacon devices the probability of deviation will be very small. In the same location, signal strength emitted by multiple beacon devices should be similar. If the difference between the signal strength exceeds a threshold value, we can identify the error and discard this RSSI error value. To solve the problem of three-dimensional space, we chose to use the compression algorithm. In our algorithm, we use the RSSI values achieved at various distances to calculate the user's position in compression algorithm.

\section{Proposed Method}

\subsection{System Architecture}

The system architecture can be divided into the beacon devices and the software application running on the user's mobile device. Beacons will periodically broadcast the RSSI signal into the positioning space and the user's mobile device will receive these signals and analyse the received data to calculate the position of the user. The system architecture is depicted in Figure 1. The user's mobile device continuously receives signals from the beacons and filters out the RSSI deviation. Then the software system will determine whether the user is in a moving state or a stationary state. The user's state is an important parameter in our system, as this parameter has a great influence on the subsequence algorithms.

Unlike previous implementations [2,3], in our system, more than one beacon device will be deployed in a corner of the room. In the experimental section of this paper, we deployed two overlapping beacons as a new beacon in every corner of the room. This method allows the received RSSI signal to be more stable, thus overcoming the disadvantages of the previous system that used BLE beacon devices. In theory, the number of beacons and the positioning error rate are inversely proportional. However, increasing the number of beacons will lead to longer computation time.

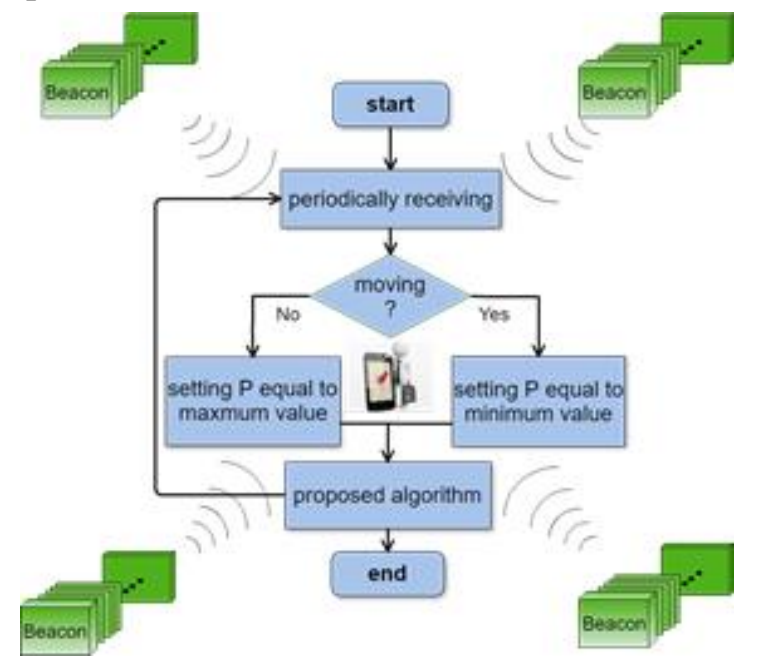

Fig. 1: The architecture of the proposed system

\subsection{Algorithm and Mathematical Model}

To build the mathematical model of proposed system, we implemented eight beacon devices in a rectangular room. Beacons are named bi, $i=1,2 \ldots 8$. Based on RSSI values obtained from these beacons we estimate the user's position. The RSSI value received at each beacon will be the average value of 10 values collected from it. When the collected data is lower than 10 values, we will use existing data to calculate the average value of RSSI. If the more than 10 values of collected data are attained then we average the 10 earliest values. The number of data may be changed according to various cases. When the user moves, the number of data will be reduced. The new received data will be compared with the average value. If the deviation does not exceed the threshold, the data will be saved and used to calculate a new average value, otherwise, this RSSI value will be discarded. 
This average of eight beacons is named ri, $i=1,2 \ldots 8$. With the overlapping beacons, we will have four new beacons named $\mathrm{Bi}, \mathrm{i}=1,2,3,4$ (in this case, two beacons will be placed at each corner). $\mathrm{Ri}$ is the RSSI value of Bi (as Eq. 1). To determine the user's location in the room, we will rely on the RSSI values obtained from the beacons. Our algorithm consists of two steps. First, we need to determine Ri, which is generated by average of signal strength ri, ri+4. The algorithm to calculate the value of Ri and filter out the RSSI deviation is as follows:

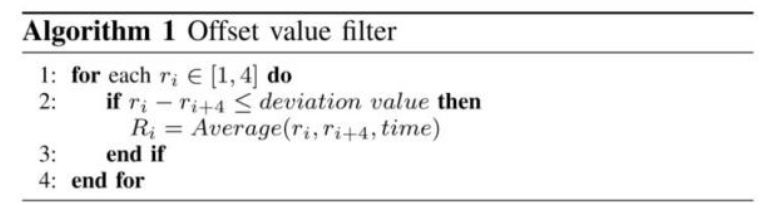

where, Average(ri, ri+4, time) is the expression to calculate the value of Ri from ri and $\mathrm{r}-\mathrm{i}+4$, time is the average number of executions. In this case, ri and ri+4 are placed in the same corner. The formula to calculate the average value is given as:

$$
\text { Average }\left(r_{i}, r_{i+4}, \text { time }\right)=\frac{\sum\left(\left(r_{i}+r_{i+4}\right) / 2\right)}{\text { time }} .
$$

Second, in Figure 2, the notation a represents the beacon with the maximum RSSI value; this value is a datum. We assume that two adjacent beacons with a are named as $b$ and $d$. Where, $b=a+1 \bmod 4$ and $d=a$ $-1 \bmod 4$. For example, assume that B1 is the beacon with maximum RSSI value, so B1 is a, B2 is b and B4 is d. We denote SRi as a relative value corresponding to data points describing the location of the user. Through this method, we convert 3D data coordinates into 2D data coordinates. Di represents the relative distance (D) between the beacon $\mathrm{Bi}$ and the user in plane space and parameter $\mathrm{P}$ will affect the accuracy of the positioning method. Utilising the trilateration, we can deduce the coordinates of $x$ and $y$. If $a+1$ is an odd number then coordinates of the $\mathrm{x}$-axis range from $\mathrm{Da}$ to $\mathrm{Dd}$ and $\mathrm{y}$-axis from $\mathrm{Da}$ to $\mathrm{Db}$. If not, coordinates of the $\mathrm{x}$-axis range from $\mathrm{Da}$ to $\mathrm{Db}$ and $\mathrm{y}$-axis from $\mathrm{Da}$ to $\mathrm{Dd}$.

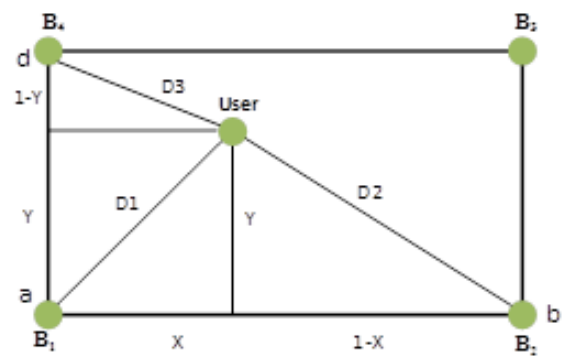

Fig. 2: The method of the positing algorithm

Assume $\mathrm{P}$ is a floating point; the formula to calculate the relative value of $\mathrm{SRj}$ is given by:

$$
S R_{j}=\left|R_{j}-\left(\max \left(R_{i}\right)+P\right)\right|, j=\overline{1 \ldots 4} .
$$

As shown in Figure 2, we can calculate the current location of the user $\mathrm{S}(\mathrm{X}, \mathrm{Y})$ using the Pythagorean triangular theorem as follows:

$$
S(X, Y)=\left\{\begin{array}{l}
X=\frac{\left(\frac{S R_{x}}{S R_{x}+S R_{y}}\right)^{2}-\left(\frac{S R_{y}}{S R_{x}+S R_{y}}\right)^{2}+1}{2}, \\
\text { if } a \bmod 2=1, x=a, y=b \text { and } z=d \\
Y=\frac{\left(\frac{S R_{x}}{S R_{x}+S R_{z}}\right)^{2}-\left(\frac{S R_{z}}{S R_{x}+S R_{z}}\right)^{2}+1}{2} \\
\text { if } a \bmod 2=0, x=a, y=d \text { and } z=b
\end{array} .\right.
$$

Figure 2 represents the algorithm of the proposed method. The relative distance between the user and beacons can be calculated by:

$$
D_{1}=\frac{S R_{a}}{S R_{a}+S R_{b}}, D_{2}=\frac{S R_{b}}{S R_{a}+S R_{b}}, D_{3}=\frac{S R_{d}}{S R_{a}+S R_{d}} .
$$




\section{Experiments}

We chose an $8.1 \times 9.9 \times 3 \mathrm{~m}$ room in which to conduct the experiment. A software application was built to calculate and display the positioning information to the user. The software is installed on a SONY XPERIA Z1 smartphone. The frequency of beacon transmission is 0.3 seconds/packet and our algorithm generates an estimated location every 0.3 seconds. In Eq. (2), the parameter $\mathrm{P}$ is an important factor that could significantly affect the results of the experiment. The size of the $\mathrm{P}$ affects user state. In this experiment, we set the value of $\mathrm{P}$ as $1,10,20,30$ and 40 and observed the highest precision of each group. We respectively allowed $20,30,40$ and $50 \mathrm{~cm}$ of positioning error. If the predicted position is out of range, then it was judged to be failure. In the moving case, we assume that a user is moving in the room with the speeds of 1, 3, 5, 7 and $9 \mathrm{~km} / \mathrm{hour}$, separately. To compare the accuracy of our algorithm with other related methods, we also implement the positioning algorithms mentioned in other papers $[2,3]$ and consider two experimental cases of stopping and moving.

\subsection{Stopping Case}

Assume user is stopped in the middle of the room. Coordinates $\mathrm{x}$ and $\mathrm{y}$ of centre is $4.05 \mathrm{~m}$ and $4.95 \mathrm{~m}$. If we allow $50 \mathrm{~cm}$ of positioning error, the legal range is $355<\mathrm{x}<455$ and $445<\mathrm{y}<545$. The obtained results are shown in Figure 3. We can see that the higher the value of $\mathrm{P}$ the greater correct estimation rate. In the first set, the accuracy at $50 \mathrm{~cm}$ can reach $98.2 \%$ and accuracy at $20 \mathrm{~cm}$ is $20.8 \%$. In the second set, accuracy at $50 \mathrm{~cm}$ is $84.4 \%$ and accuracy at $20 \mathrm{~cm}$ is $17.5 \%$. Using the methods from previous papers [2,3] only one beacon in each corner. Hence, RSSI in these papers is not reliable. In Figure 4, we compare the correct estimation rate (CER) of our paper with the results from these previous papers $[2,3]$. The results shown that our method consistently achieves the highest CER.

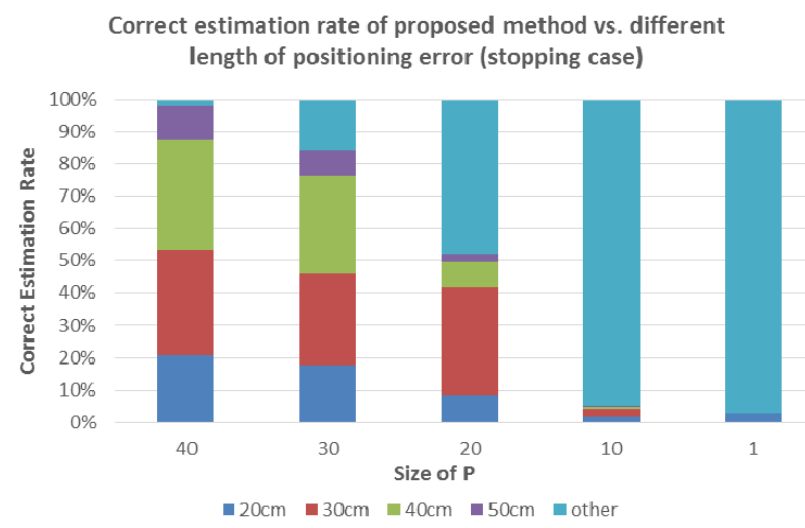

(a)

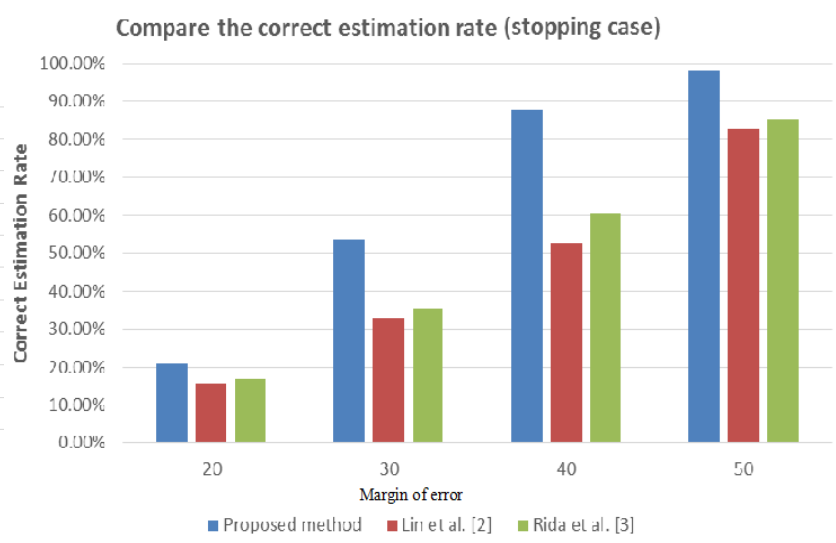

(b)

Fig. 3: Analysis of the correct estimation rate of the proposed method in stopping case. (a) The correct estimation rate of proposed method vs. different length of positioning error as the size of $\mathrm{P}$ changes. (b) Comparison of proposed method, Lin et al. [2] and Rida et al. [3] in stopping case.

\subsection{Moving Case}

Correct estimation rate of proposed method vs. different speed of User

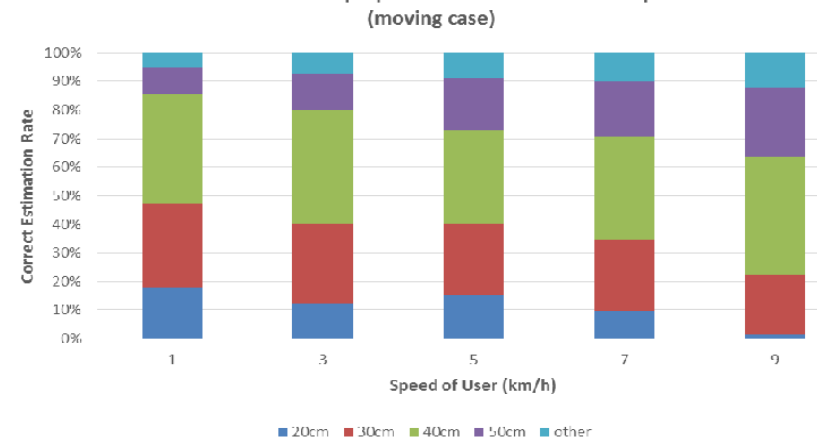

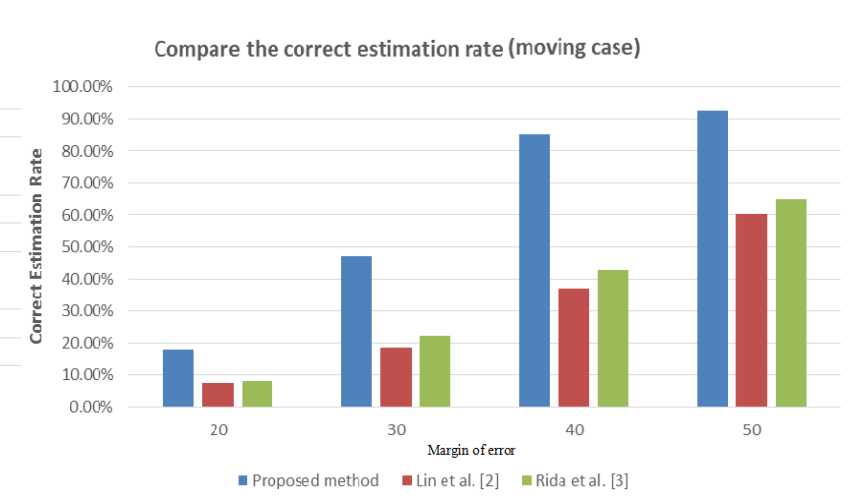

Fig. 4: Analysis of the correct estimation rate of the proposed method in moving case. (a) The correct estimation rate of proposed method with different lengths of positioning error in moving case. (b) Comparison of the proposed method,

Lin et al. [2] and Rida et al. [3] in moving case. 
Assume that the user is moving indoors. We also consider the influence of parameter $\mathrm{P}$ to the accuracy of the positioning method. In Figure 4(a), we assume $P$ is 1 and speeds of the user are 1, 3, 5, 7 and $9 \mathrm{~km} / \mathrm{h}$, separately. With the speed of $1 \mathrm{~km} / \mathrm{h}$, accuracy at $50 \mathrm{~cm}$ can reach $94.6 \%$ and accuracy at $20 \mathrm{~cm}$ can reach nearly $18 \%$. In our paper, filter out the deviation of the RSSI values; however, this method is not applied in the previous papers [2,3]. As the user moves, the signal stability is more important. In Figure 4(b), we compare the CER of our proposed method with results from papers [2] and [3]. Our method achieved the best accuracy.

\section{Conclusion}

In this paper, we proposed an IPS using BLE beacon devices. Our system uses multiple beacons devices instead of a single beacon device in each corner of a positioning plane, thereby decrease the fluctuation of received RSSI signal and enhancing the CER. Our mathematical model and implemented results show that our system achieves higher positioning accuracy than other previously reported methods.

\section{Acknowledgement}

We thank the Ministry of Science and Technology of Taiwan for supports of this project under grant number MOST 105-2221-E-035-065 and MOST105-2622-E-035-008-CC3. We thank co-authors and reviewers for their valuable opinions.

\section{References}

[1] S. Kajioka, T. Mori, T. Uchiya, I. Takumi, and H. Matsuo. Experiment of Indoor Position Presumption based on RSSI of Bluetooth le Beacon. Global Conference on Consumer Electronics. 2014, pp. 337-339.

[2] X. Lin, T. Ho, C. Fang, Z. Yen, B. Yang and F. Lai. A Mobile Indoor Positioning System based on ibeacon Technology. Annual International Conference of the IEEE Engineering in Medicine and Biology Society. 2015, pp. 4970-4973.

[3] M. Rida, F. Liu, Y. Jadi, A. Algawhari and A. Askourih. Indoor Location Position based on Bluetooth Signal Strength. International Conference on Information Science and Control Engineering. 2015, pp. 769-773.

[4] N. Mahiddin, N. Safie, E. Nadia, S. Safei and E. Fadzli. Indoor Position Detection using Wi-Fi and Trilateration Technique. International Conference on Informatics and Applications. 2012, pp.362-366.

[5] L. Png, L. Chen, S. Liu, W. Peh. An Arduino-based Indoor Positioning System (IPS) using Visible Light Communication and Ultrasound. IEEE International Conference on Consumer Electronics Taiwan. 2014, pp. 217218. 